\title{
An ultra-high input impedance ECG amplifier for long-term monitoring of athletes
}

This article was published in the following Dove Press journal:

Medical Devices: Evidence and Research

2 July 2010

Number of times this article has been viewed

\author{
Gaetano Gargiulo',2 \\ Paolo Bifulco' \\ Mario Cesarelli' \\ Mariano Ruffo' \\ Maria Romano ${ }^{1}$ \\ Rafael A Calvo 2 \\ Craig Jin² \\ André van Schaik ${ }^{2}$ \\ 'Dipartimento di Ingegneria \\ Elettronica, Biomedica e delle \\ Telecomunicazioni, Federico II \\ University of Naples, Italy; ${ }^{2} \mathrm{School}$ \\ of Electrical and Information \\ Engineering, University of Sydney, \\ Australia
}

Correspondence: Gaetano Gargiulo School of Electrical and Information Engineering, University of Sydney, Sydney, NSW 2006, Australia Email gaetano@ee.usyd.edu.au

\begin{abstract}
We present a new, low-power electrocardiogram (ECG) recording system with an ultra-high input impedance that enables the use of long-lasting, dry electrodes. The system incorporates a low-power Bluetooth module for wireless connectivity and is designed to be suitable for long-term monitoring during daily activities. The new system using dry electrodes was compared with a clinically approved ECG reference system using gelled $\mathrm{Ag} / \mathrm{AgCl}$ electrodes and performance was found to be equivalent. In addition, the system was used to monitor an athlete during several physical tasks, and a good quality ECG was obtained in all cases, including when the athlete was totally submerged in fresh water.

Keywords: bioelectric potentials, biomedical electrodes, ECG monitoring
\end{abstract}

\section{Introduction}

The current trend in patient monitoring and diagnosis is towards an increased usage of portable or even wearable devices. These personal devices will improve follow-up of chronic patients, diagnosis, and identification of paroxysmal symptoms, monitoring of the elderly, and evaluations of human performance in particular conditions and/or environments.

Cardiology is one branch of medical science that could clearly benefit from the availability of long-term monitoring. It is well known that morphologic changes or the presence of various arrhythmias in the long-term electrocardiogram (ECG) have a strong correlation with heart disease and coronary artery disease. ${ }^{1}$ Also, the recurrence of atrial fibrillation after ablation is not uncommon and this can only be tracked using long-term ECG monitoring. ${ }^{2}$

Long-term ECG monitoring in cardiology is not only useful for follow-up of patients when their pathologic status is already known, but also for monitoring of the athlete during exercise. The possibility that young, highly trained or even professional athletes may harbor potentially lethal heart disease or be susceptible to sudden death under a variety of circumstances seems counterintuitive. Nevertheless, such sudden cardiac catastrophes do occur, usually in the absence of prior symptoms, and have a considerable emotional and social impact on the community. ${ }^{3}$ As a result of the ECG screening programs for athletes which are now compulsory in many countries, it is now known that many of these sudden deaths are due to a syndrome called "athlete's heart". ${ }^{3-5}$ This syndrome may be associated with rhythm and conduction alterations, morphologic changes in the QRS complex on the ECG, and pathologic repolarization abnormalities. ${ }^{5}$ However, it is broadly accepted that the standard 12-lead ambulatory ECG is submit your manuscript $\mid$ www.dovepress.com

Dovepress

9321
Medical Devices: Evidence and Research 2010:3 I-9

(C) 2010 Gargiulo et al, publisher and licensee Dove Medical Press Ltd. This is an Open Access article which permits unrestricted noncommercial use, provided the original work is properly cited. 
not enough to clarify the origin of an ECG abnormality, especially if it is triggered by exercise. This creates a need for a system that is able to record the ECG during exercise reliably and without interference.

For standard ECG measurements, electrodes are attached to the patient's skin after skin preparation, which includes cleaning, shaving, mechanical abrasion to remove dead skin, and moistening. A layer of electrically conductive gel is applied in between the skin and the electrodes to reduce the contact impedance. ${ }^{6}$ However, in these so-called wet electrodes, the electrolytic gel dehydrates over time, which reduces the quality of the recorded signals. In addition, the gel might leak, particularly when an athlete is sweating, which could electrically short the recording sites. This is an even bigger problem for monitoring athletes immersed in water. Securing the wet electrodes in place is also complicated, because the electrodes cannot be glued directly to the skin due to the presence of the gel. The use of dry or insulating electrodes avoids these problems. ${ }^{7}$

Conductive rubber has several advantages when used in dry electrodes. They become durable, washable, and reusable, the carbon and silicon materials are biocompatible, they provide a smoother and more uniform contact surface with the skin, and are thin, flexible, and easily applied to a variety of substrates. ${ }^{8,9}$ However, they also have a much higher impedance compared with conventional wet electrodes. ${ }^{10}$

Alternatively, textile electrodes, which can be embedded in clothes, can be used as dry electrodes. ${ }^{11,12}$ These offer a high degree of patient independence, freedom of movement, and are suitable for long-term monitoring purposes. Textile electrodes are typically made of synthetic materials that withstand abrasion very well, do not irritate the skin, and are lightweight and washable. A major drawback is the high contact impedance of these electrodes, typically $1-5 \mathrm{M} \Omega / \mathrm{cm}^{2}$, compared with a $10 \mathrm{k} \Omega / \mathrm{cm}^{2}$ impedance for the disposable $\mathrm{Ag} / \mathrm{AgCl}$ electrodes. ${ }^{13,14}$

In fact, high electrode impedance is a common issue with all types of dry electrodes. Therefore, to use these electrodes, they must be connected to instrumentation amplifiers with extremely high input impedances. ${ }^{15}$ One solution is to buffer the electrode signal directly at the electrode to provide impedance conversion; ${ }^{15,16}$ this type of assembly is referred to as an active electrode. This approach requires that some rigid circuitry is fixed on the electrode, increasing the size of the electrode, and resulting in an inflexible electrode in the case of textile electrodes.
Here we propose a biopotential amplifier with an ultra-high input impedance for use with dry electrodes. The design uses two commercial integrated circuits and can thus be readily replicated by other researchers in the field. Laboratory and clinical tests demonstrate that the system is able to acquire the ECG of subjects, and reliable monitoring can be performed for at least 24 hours. The device is shown to work even when the subject is fully immersed in water.

\section{Methods \\ Biopotential amplifier}

We used the Burr-Brown INA116 as the implementation amplifier because it currently has the highest input impedance available $\left(10^{15} \Omega\right)$. It has a maximum input bias current of $3 \mathrm{fA}$ and an input current noise of $0.1 \mathrm{fA} / \sqrt{ } \mathrm{Hz}$. This integrated circuit also offers integrated active input shielding which is used to shield the electrodes. Furthermore, it features built-in electrostatic discharge and overvoltage protection. ${ }^{17}$

Figure 1 shows the implementation of the front end of the device. The component values used for the ECG monitor are given in Table 1. The INA116 is designed to work with a $9 \mathrm{~V}$ dual power supply, but due to the very low bandwidth and the small amplitude of the biologic signals we intended to measure, we were able to use it with a single-ended voltage supply as low as $2 \mathrm{~V}$. The amplifier gain can be configured from $700 \mathrm{~V} / \mathrm{V}$ up to $1 \mathrm{kV} / \mathrm{V}$, depending on the application, by varying the value of the resistor RG2 from $7.5 \mathrm{k} \Omega$ up to $5.7 \mathrm{k} \Omega$; these values take into account the voltage drop operated by the passive high pass cell (RLpass - Clpass). In order to allow a larger signal excursion, the lower value in athlete monitoring was always used.

The circuit is preferably operated from a single battery. A virtual signal ground is derived from the battery using a voltage divider (R5/R6). The virtual ground is buffered to provide a driven ground connection for the right leg via a calibrated coupling impedance $\mathrm{R}$ couple.

The analog front end contains a low pass filter and a high pass filter to limit the bandwidth of the recorded signal. The cutoff frequency for the high pass filter is tunable from near direct current up to $5 \mathrm{~Hz}$, by changing the value of CHpass while RHpass is kept fixed at $390 \mathrm{k} \Omega$. The cutoff frequency of the low pass filter is regulated by tuning CLpass while keeping RLpass fixed at $1 \mathrm{M} \Omega$. Using commercially available precision components, we implemented a bandwidth of $0.38-44 \mathrm{~Hz}( \pm 5 \%)$, suitable for most ECG and EEG applications. ${ }^{18}$

The second stage of amplification and the driven ground are implemented using a low-power, precision operational 


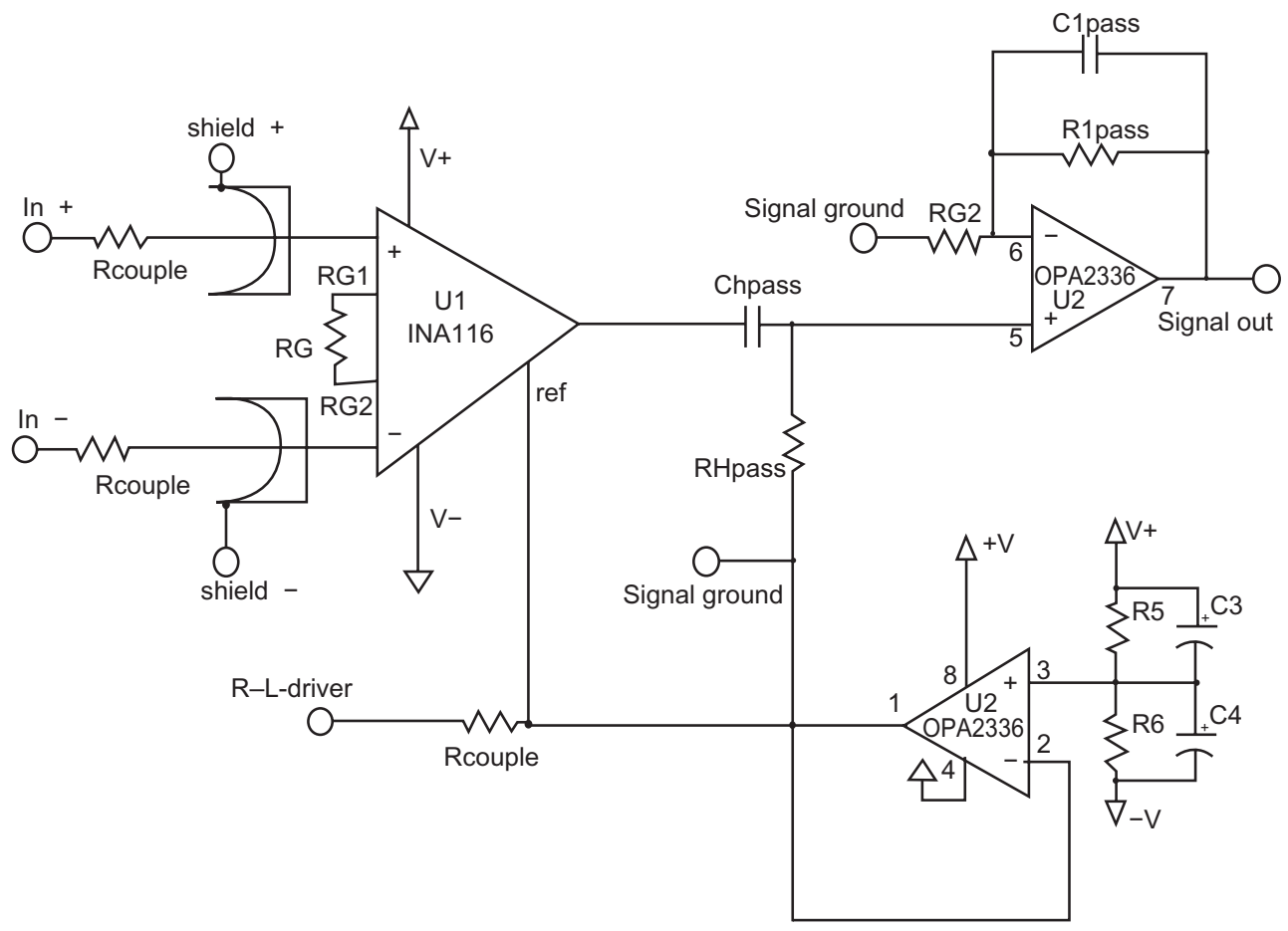

Figure I Schematics of the biopotential amplifier

amplifier, ie, the Burr-Brown OPA2336. The second stage of amplification, together with the band pass filtering provides sufficient gain and high frequency suppression to feed the ADC converter directly.

It is worthwhile to highlight that in order to minimize noise capturing and to avoid introducing imbalance at the input of the bioamplifier, the tolerance of the R couple impedance is critical. Hence it is necessary to choose between the low tolerance ones.

For data transmission we used the Corscience BluesenseAD Bluetooth transceiver, which fulfills the EN 60601-1-2 medical electrical equipment standard. ${ }^{19}$ It has an integrated 12-bit ADC and is designed to accept input signals up to $3.6 \mathrm{Vpp}$. We chose to operate our analog front end with a $4.8 \mathrm{~V}$ supply, and a limiting circuit has been added to the input of the ADC to protect the circuit. Figure 2 shows the BlueSenseAD schematic, including the necessary voltage

Table I Component values

\begin{tabular}{llll}
\hline RHpass & $\mathbf{3 9 0} \mathbf{k} \Omega$ & CHpass & $\mathbf{I} \boldsymbol{\mu F}$ \\
\hline RLpass & $\mathrm{I} \mathrm{M} \Omega$ & CLpass & $3.3 \mathrm{nF}$ \\
R5, R6 & $170,102 \mathrm{k} \Omega$ & $\mathrm{C} 3, \mathrm{C} 4$ & $\mathrm{I} \mu \mathrm{F}$ \\
RG & $10 \mathrm{k} \Omega$ & Rcouple & $100 \mathrm{k} \Omega$ \\
RG2 & $5.7-7.5 \mathrm{k} \Omega$ & & \\
CbI, Cb3, Cb5 & $10 \mu \mathrm{F}$ & $\mathrm{Cb} 2, \mathrm{Cb} 4, \mathrm{Cb} 6$ & $100 \mathrm{nF}$ \\
\hline
\end{tabular}

regulators at $3.3 \mathrm{~V}$ for the digital circuitry and $3.6 \mathrm{~V}$ for the analog. The BlueSenseAD can operate in two modalities, ie, standard mode and SNIFF mode. In standard mode the transreceiver will consume $<160 \mathrm{~mW}$, while in SNIFF mode the power consumption drops down to $<30 \mathrm{~mW}$. However, in SNIFF mode, the data rate drops to $10 \mathrm{Kbs}$; such a data rate ensures a total sample rate of $500 \mathrm{~Hz}$ that is adequate for our application. ${ }^{20}$

We have selected the gains to be such that the ECG signal uses most of the ADC dynamic range (at least 2000 levels), while being small enough to ensure that only large artifacts are clipped by the limiting circuitry. The limiting circuitry is realized using a signal diode (1N4148, D1 in Figure 2) with the cathode connection to the $3.3 \mathrm{~V}$ supply rail, thus clipping signals larger than $3.9 \mathrm{~V}$.

The ultra-high input impedance of the bioamplifier allows us to use a new kind of dry passive electrode. Moreover, it makes the recorded electrode signal immune to variation or imbalance in the contact impedance between electrodes. In fact, our system allows mixing of different electrode types, ie, some dry and some wet.

\section{Dry electrodes}

The dry electrodes are made from commercially available $1.5 \mathrm{~mm}$ thick silicon conductive rubber, shaped in several 


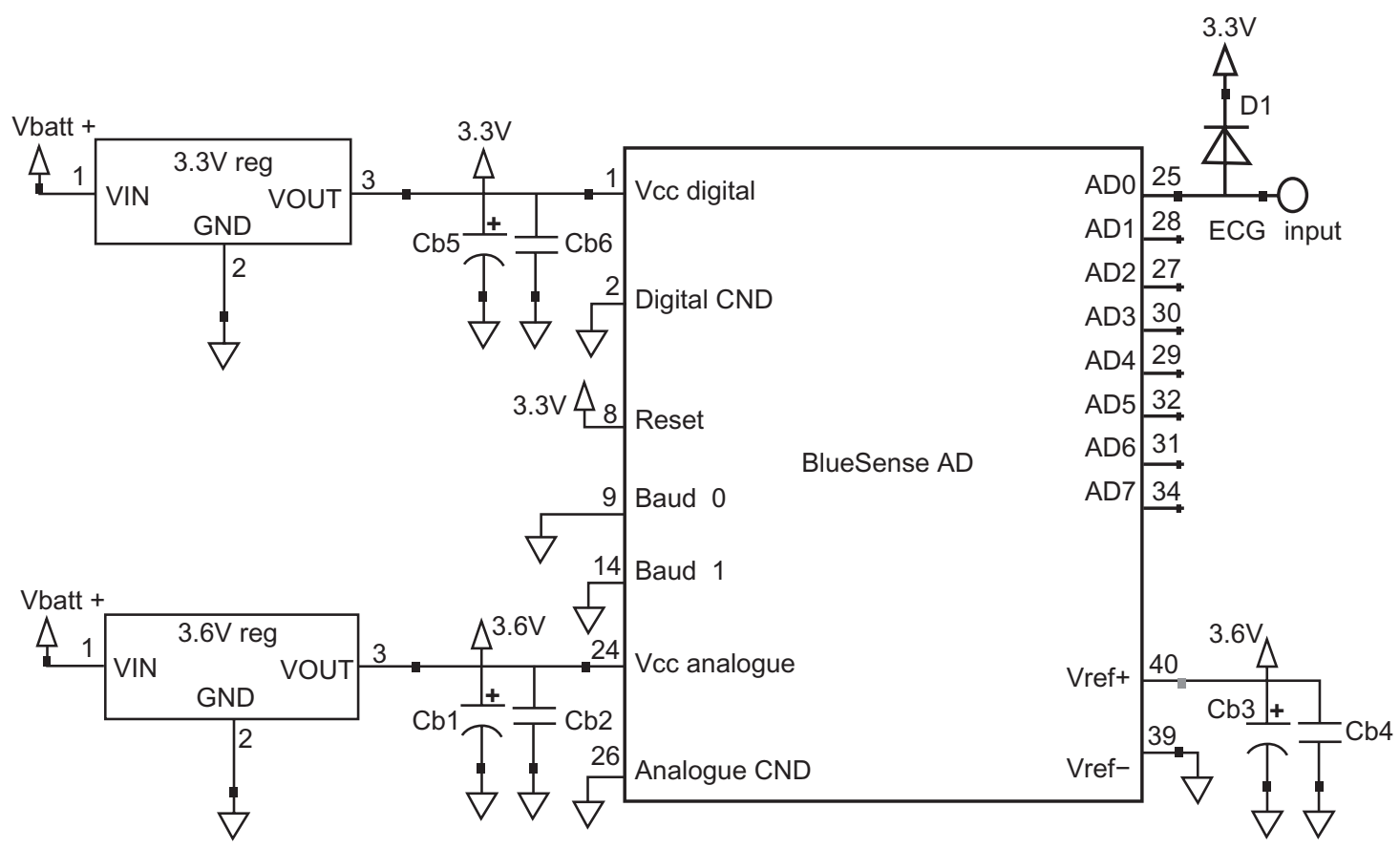

Figure 2 Schematics of the BlueSenseAD connections.

forms depending on the specific application, including discs of $8 \mathrm{~mm}$ diameter. ${ }^{19,21}$ This conductive rubber has been used in stimulating electrodes for decades, but not for recording electrodes because of its intrinsic high ohmic resistance. ${ }^{10,22}$

Figure 3 shows an illustrative diagram of the dry electrode. In practice, an additional layer of insulation is added to cover the shielding plate to protect it from contact. The active side of the electrode is capacitively coupled through a layer of insulating silicon rubber with a metal shield connected to the active guard shield. The measured impedance of the electrodes at $10 \mathrm{~Hz}$ is greater than $20 \mathrm{M} \Omega$.

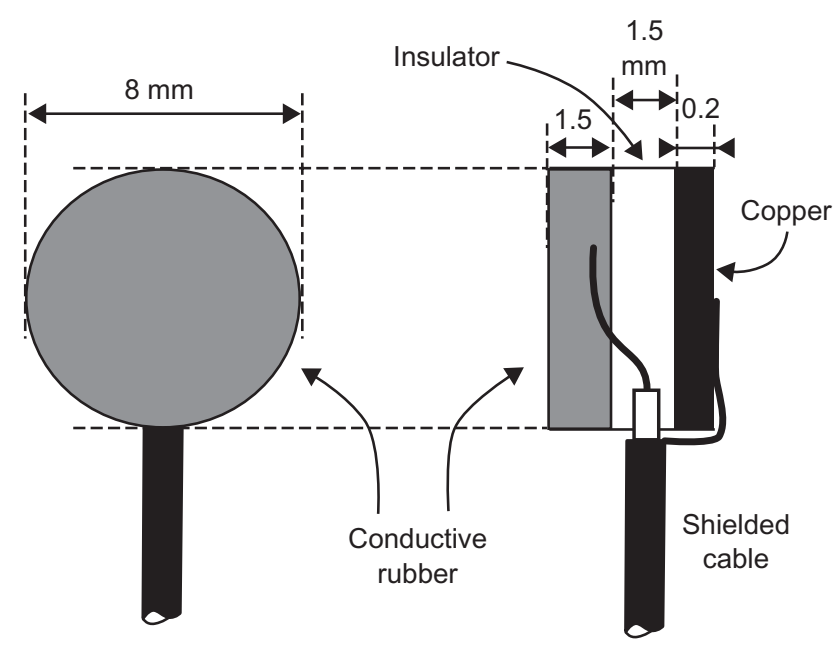

Figure 3 Front view and cross section of the conductive rubber electrode.

\section{Results}

\section{Bench testing}

Bench tests of the biopotential amplifier were carried out using a Fluke MEDSIM 300B ECG signal generator. Table 2 shows a summary of the measured specifications of the system. The power consumption of the system is less than $5 \mathrm{~mA}$ at $4.8 \mathrm{~V}$. The leakage current of the biopotential amplifier was measured according to the EN 60601-1-2 standard by using a Metron QA 80 safety analyzer. Our measurements show that the leakage current on each lead is less than $1 \mu \mathrm{A}$.

Internally generated noise was evaluated while shorting the input electrodes. ${ }^{23}$ Data were recorded using a sample rate of $2 \mathrm{kHz}$ with $1 \mathrm{~m}$ in length shielded leads terminated with electrodes as depicted in Figure 3. The two electrodes were kept in contact with each other using a nonmetallic clamp. The measured total input referred noise in the frequency band $0.1-100 \mathrm{~Hz}$ was $0.660 \mu \mathrm{Vrms}$. The input referred noise

Table 2 System specifications

\begin{tabular}{ll}
\hline ECG Vdd & $\mathbf{4 . 8 ~ V}$ \\
\hline ECG power consumption & $<5 \mathrm{~mW}$ \\
Electrode leakage current & $<\mathrm{I} \mathrm{\mu \textrm {A }}$ \\
Gain & $90 \mathrm{~dB}$ \\
Input referred noise $(0.1-100 \mathrm{~Hz})$ & $0.660 \mu \mathrm{Vrms}$ \\
Bandwidth & $0.38-44 \mathrm{~Hz}( \pm 5 \%)$ \\
BlueSenseAD power consumption & $<160 \mathrm{~mW} /<30 \mathrm{~mW}$ \\
\hline
\end{tabular}




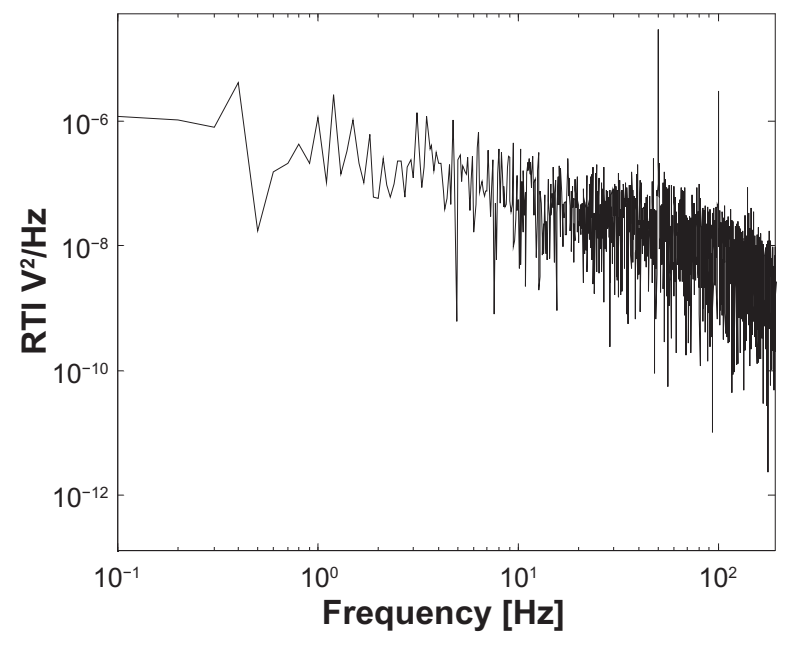

Figure 4 Input referred noise power spectral density of the biopotential amplifier and its dry electrodes.

as a function of the frequency of the bioamplifier and the electrodes, as shown in Figure 4.

Figure 5 shows the gain versus frequency of the biopotential amplifier as measured. As expected, the system displays band-pass behavior with a bandwidth in the range of $0.38-44 \mathrm{~Hz}( \pm 5 \%)$, given the precision of the passive components.

\section{ECG comparison}

Figure 6 shows two simultaneous ECG recordings (lead I) of a subject immediately after fixing the electrodes onto the subject's forearms. The top trace was recorded with our system whereas the bottom trace was recorded using wet $\mathrm{Ag} / \mathrm{AgCl}$ electrodes and a commercial biopotential amplifier (g.Bsamp). No skin preparation was used for the dry electrodes, but preparation was necessary for the wet electrodes. Recorded data were compared sample-by-sample, and the correlation between the two recordings was always larger than 0.96 .

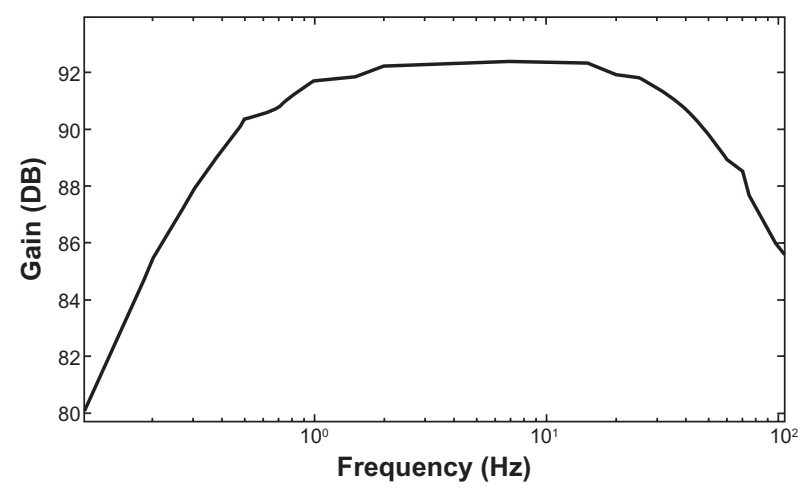

Figure 5 Gain of the biopotential amplifier as a function of frequency.
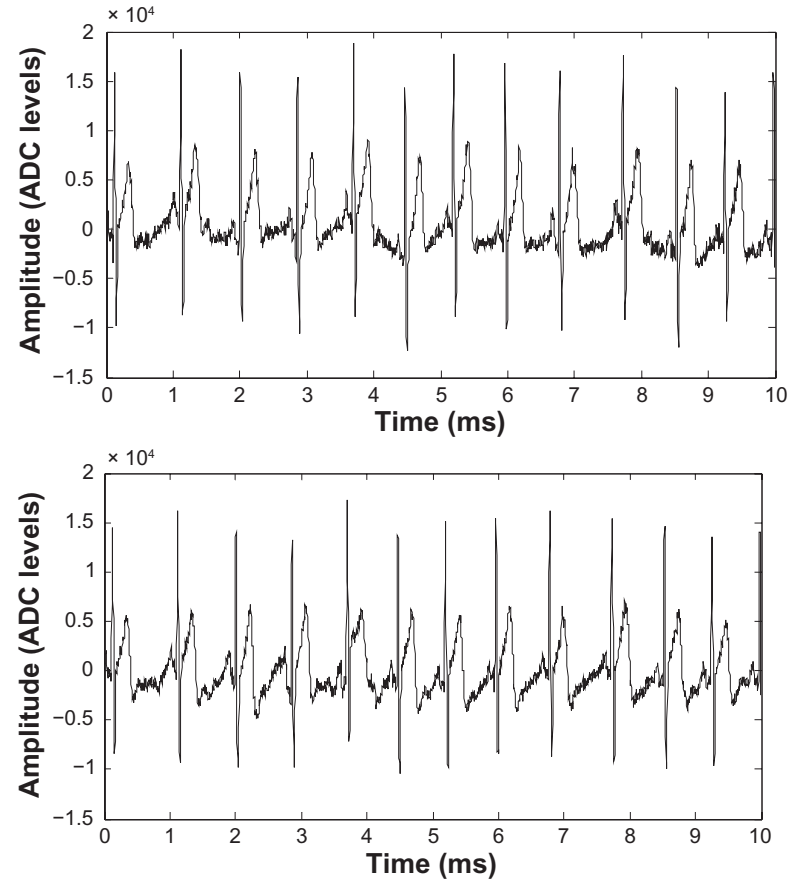

Figure 6 Direct comparison of an ECG recording with the proposed system (top) and a clinically approved system (g.Bsamp) using wet $\mathrm{Ag} / \mathrm{AgCl}$ electrodes.

Figure 7 shows the same two simultaneous lead I ECG recordings after the electrodes were left in place on the subject for 48 hours. The ECG recorded with the wet electrodes and the g.Bsamp shows large artifacts, while the ECG from the dry system only showed a slight increase in baseline variation and in high frequency noise.

\section{Exercise monitoring}

The advantages of wireless monitoring using the proposed complete device are particularly strong when performing long-term monitoring during activities of daily living, or when monitoring athletes during exercise. We tested our device on a subject undertaking certain typical body-building exercises. In order to keep a stable and comfortable contact between the skin and our dry electrode,

We used an elastic chest strap. The strap was comfortable to wear and does not constrain the movement of the subject. Electrodes are placed on the anterior side of the torso below the chest muscles. The prototype enclosed in a lightweight plastic box (measuring $40 \times 60 \times 20 \mathrm{~mm}$ ) also containing the battery pack was secured on the belt. The device was comfortable to wear and not obstructive during the physical task.

Figure 8 shows an example of the ECG data recorded on our subject during resting. The sample rate for all the presented plots was set to the standard value of $200 \mathrm{~Hz}$ because the data are clearly readable without any need for 

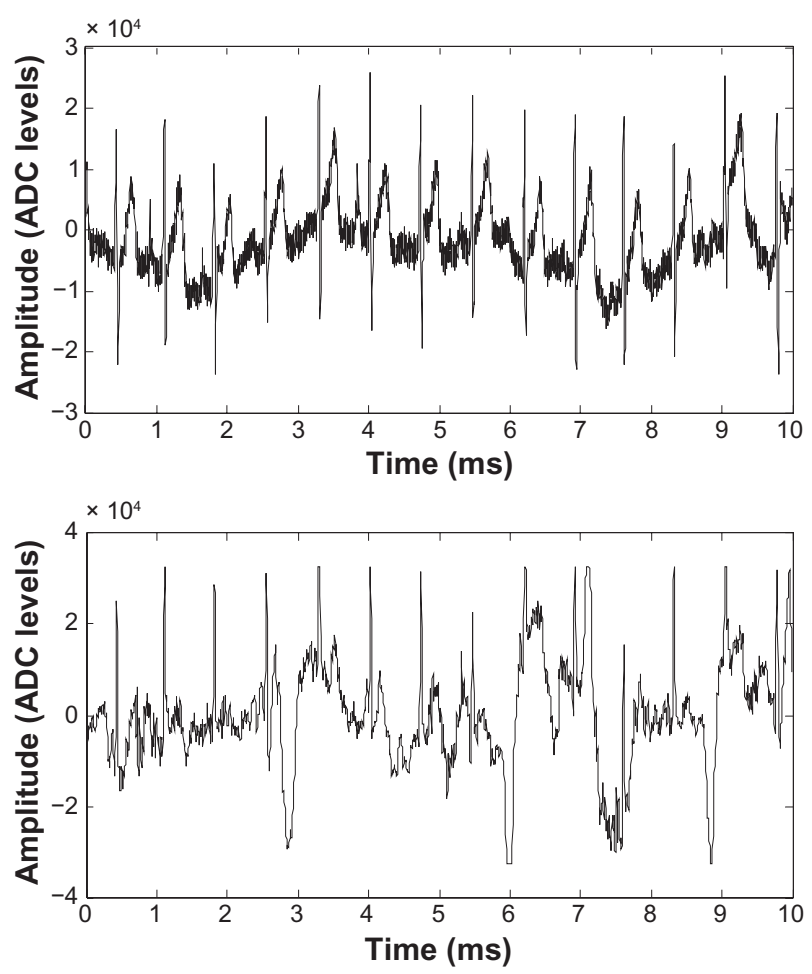

Figure 7 Direct comparison of an ECG recording with the proposed system (top) and a clinically approved system (g.Bsamp) using wet $\mathrm{Ag} / \mathrm{AgCl}$ electrodes after 48 hours.

post-processing. The medical staff who helped us to analyze the data ensured that the ECG signals were within normal range.

Figure 9 shows an example of raw ECG data (top trace) recorded during a squatting exercise session. It is possible to observe the start of exercise around the seventh second; the ECG baseline moves slightly and shows more high frequency content, ie, electromyographic (EMG) artifact, because of the slight involvement of the chest muscles in this exercise. The bottom trace in Figure 9 shows the same data after band pass filtering in the software to reduce the bandwidth to $2-30 \mathrm{~Hz}$. This removes the DC value of the ADC output and the baseline variation during exercise, and decreases the

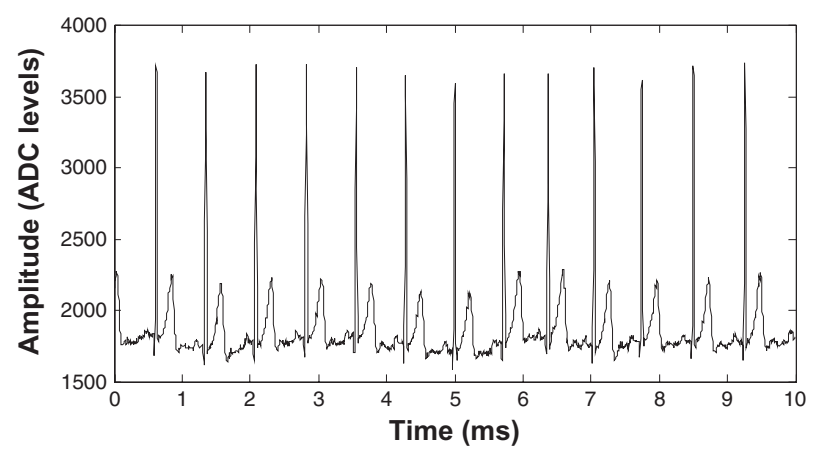

Figure 8 ECG recorded with our system during rest.
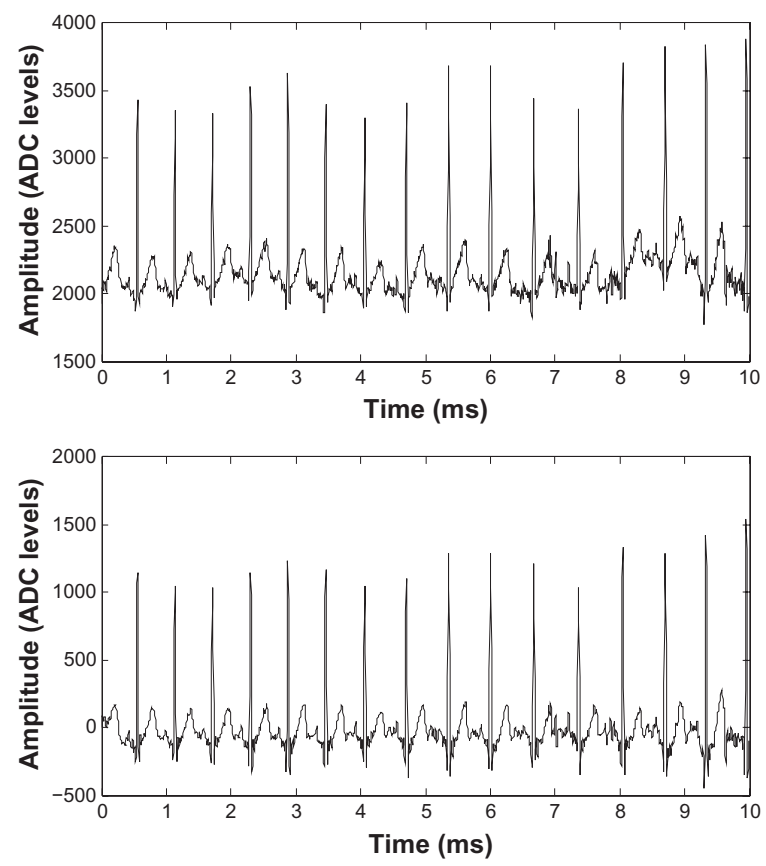

Figure 9 ECG signals recorded during a squatting exercise. Top: raw data, bottom: band pass filtered data.

high frequency noise generated by muscle contraction. The latter becomes more important as the chest muscles become more involved in exercises such as a bench-press or a full body pullup.

Figure 10 shows an extract of the raw data (top) and post-filtered data (bottom) recorded during a bench-press
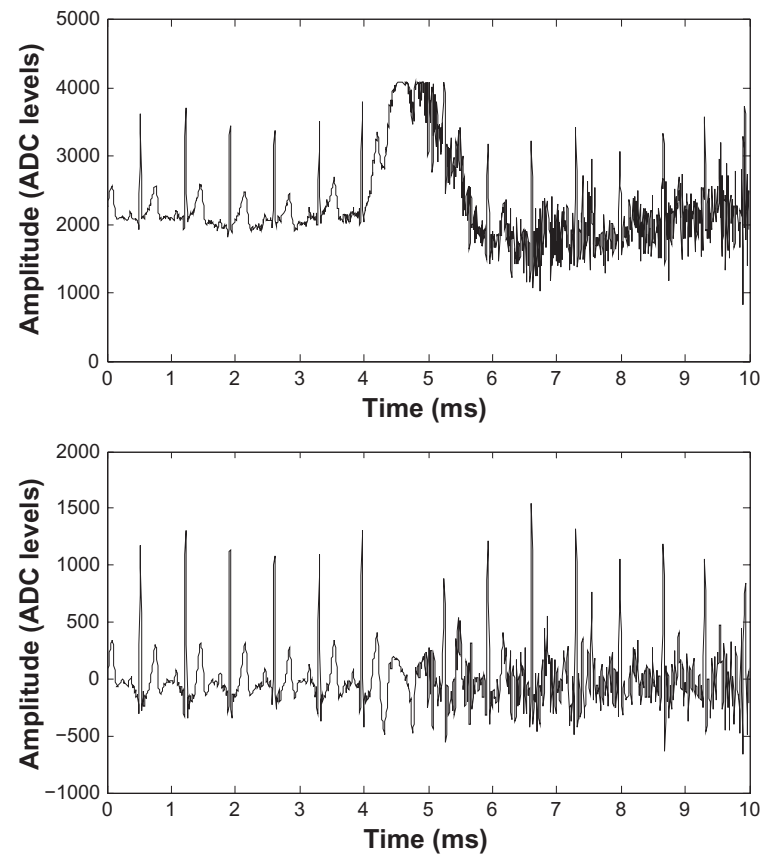

Figure 10 ECG signals recorded during a bench press exercise. Top: raw data, bottom: band pass filtered data. 
session. EMG artifacts polluting the ECG signal for this exercise can be observed starting around the fourth second. In the post-processed data, the effect of the EMG artifacts is reduced and the heart beats are clearly visible. A large baseline variation is visible around the fifth second in the top trace, causing the system to saturate and one heart beat is lost as a consequence. Figure 11 shows results for the pullup exercise session. Even larger EMG artifacts and baseline variations are present during this exercise, but post-filtering is still able to recover nearly all heart beats.

\section{Underwater monitoring}

Even though our rubber electrodes are designed to operate in a dry environment, they also work in a wet environment where gel is applied. In fact they can even work when submerged in water. This enables the device to be used for monitoring athletes in wet environments and even swimmers. Figure 12 shows a sample of the data recorded from a subject totally submerged in fresh water. No special skin preparation was used and no waterproofing was done at the electrode level. In this experiment we did not use the Bluetooth interface but instead a simple self-powered 12 bit USB module was used for ADC. The module was enclosed, together with the ECG front end, into a waterproof (IP68 compliant) box worn by the subject. This was connected via a USB cable to a computer several meters away from the water.
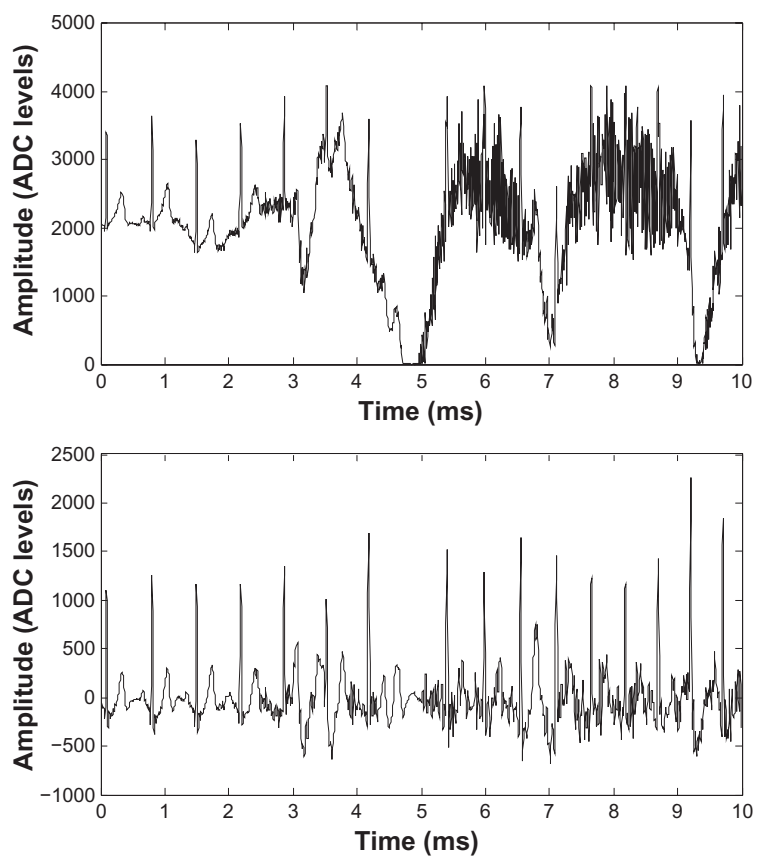

Figure I I ECG signals recorded during a pullup exercise. Top: raw data, bottom: band pass filtered data.
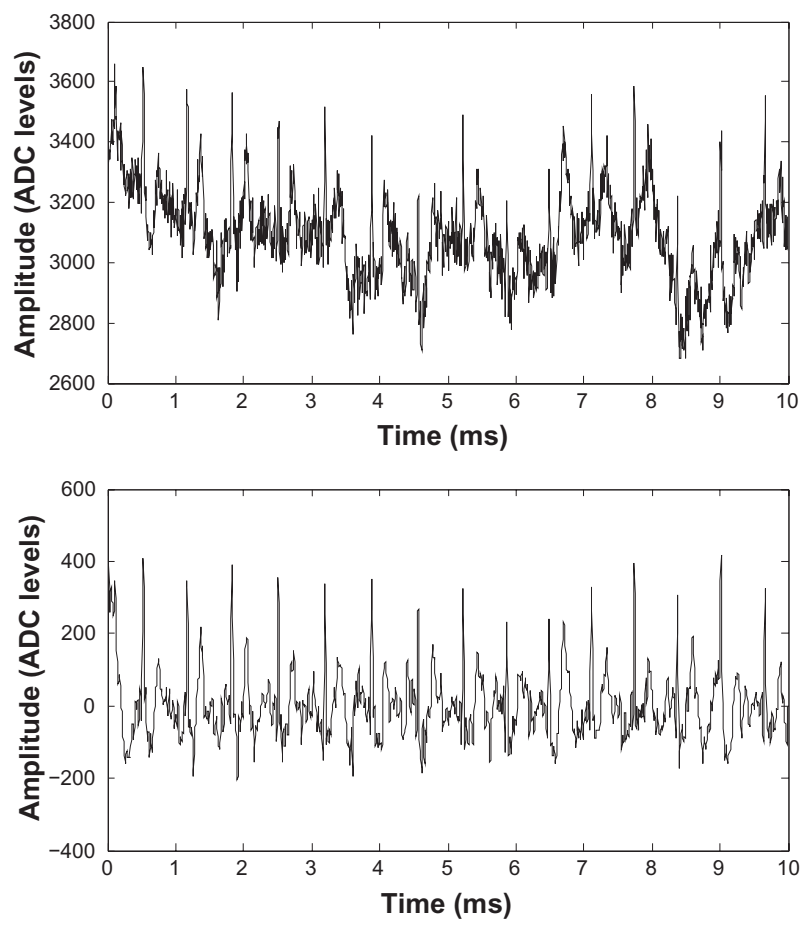

Figure 12 Underwater ECG recording. Top: raw data, bottom: band pass filtered data.

Again the top trace of Figure 12 shows strong EMG artifacts, which are the result of the subject using his arms and chest muscles to keep himself totally submerged. Postprocessing of the data by band pass filtering recovers the heart beats cleanly.

\section{Monitoring using textile electrodes}

Rather than using rubber electrodes held in place by an elastic strap, it is also possible to use textile electrodes with our amplifier. Figure 13 shows a sample of ECG data recorded using textile electrodes (by Textronics http://www.textronicsinc. $\mathrm{com} /$ ) knitted into the fabric of an adherent gym garment. Electrodes were placed on the anterior part of the torso (just below the chest musculature). For this test, the garment was worn continually for 24 hours. The top panel in Figure 13 shows raw data recorded after 24 hours, while the bottom panel shows the same sample of data after band pass filtering $(2 \div 30 \mathrm{~Hz})$, to remove chest muscle EMG artifacts and breathing artifacts.

\section{Discussion}

An ultra-high input impedance biopotential amplifier together with dry electrodes is presented. A comparative test with a clinically approved monitoring device shows performance equivalent to the systems used in a standard laboratory setting. Furthermore, our experiments show that this system 

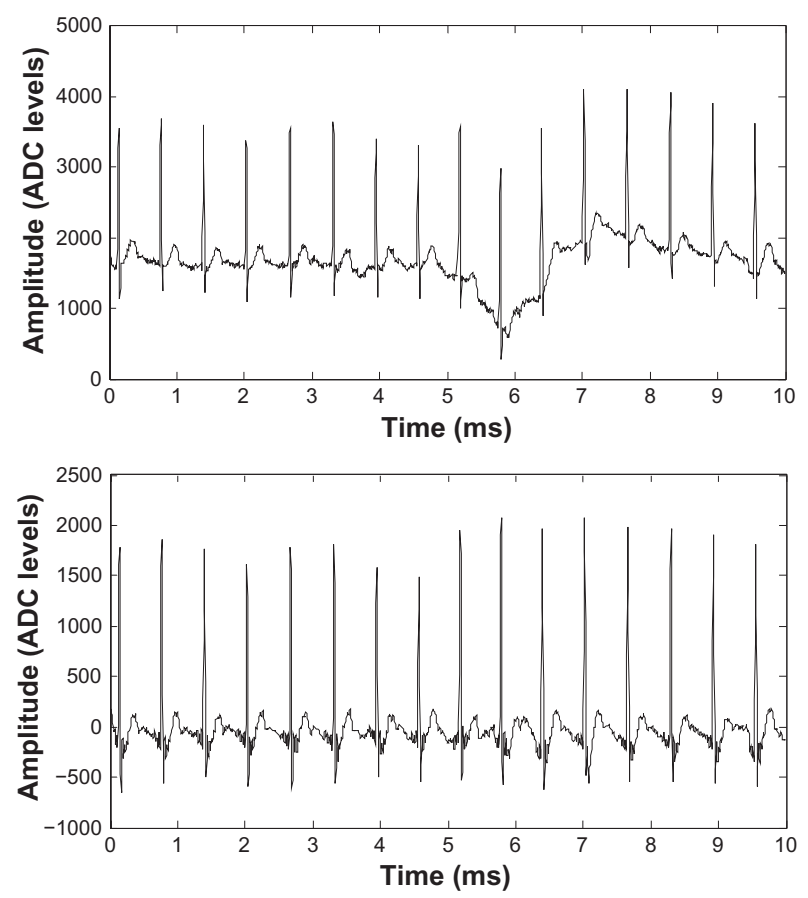

Figure 13 ECG signals recorded using textile electrodes after 24 hours of wearing. Top: raw data, bottom: band pass filtered data.

can be used to monitor the ECG during physical exercise, even when the athlete is submerged in water.

The circuit uses only two integrated circuits and few passive components and can be easily miniaturized. However, despite its apparent simplicity, the printed circuit board must be laid out and constructed with care to take full advantage of the INA116's ultra-high input impedance. These precautions include keeping all surfaces of the printed circuit board, connectors, and components free of contaminants, such as smoke particles, dust, and humidity. Usage of a Teflonprinted circuit board material instead of the more common glass-epoxy type is recommended. Digital conversion and secure wireless communication is achieved using a Bluetooth module which fulfills the EN 60601-1-2 medical electrical equipment standard.

The whole system (including the Bluetooth module radio) only consumes $33 \mathrm{~mA}$ of supply current operating with a $4.8 \mathrm{~V}$ power supply for which only $1 \mathrm{~mA}$ is needed for the analog part. This allows the device to be operated continuously for more than 12 hours on a standard $2400 \mathrm{mAh}$ lightweight mobile phone size rechargeable $4.8 \mathrm{~V}$ battery pack.

In conclusion, this biopotential amplifier could be successfully used in standard clinical monitoring applications and in many new and/or unusual applications, such as ambulatory monitoring of patients, sports medicine, underwater monitoring, and monitoring of heart rate whether the patient is standing, sitting or lying.

\section{Acknowledgments}

This work was supported by a University of Sydney research grant. The authors thank Burr-Brown Semiconductor, Maxim Semiconductor, and Samtec, for kindly providing samples for this research.

\section{Disclosure}

The authors report no conflict of interest in this work.

\section{References}

1. Zheng ZJ, Croft JB, Giles WH. State-specific mortality from sudden cardiac death. MMWR Morb Mortal Wkly Rep. 2002;5:51:123-126.

2. Hindricks G, Piorkowsky C, Tanner H. Perception of atrial fibrillation before and after radiofrequency catheter ablation: Relevance of asymptomatic arrhythmia recurrence. Circulation. 2005;112:307-313.

3. Maron BJ. Sudden death in young athletes. Lessons from the Hank Gathers affair. N Engl J Med. 1993;329:55-57.

4. Basilico FC. Cardiovascular disease in athletes. Am J Sports Med. 1999; 27:109-121.

5. Fagard F. Athlete's heart. Heart. 2003;89:1455-1461.

6. Webster JG, editor. Medical Instrumentation Application and Design. 3rd ed. New York, NY: John Wiley \& Sons; 1998.

7. Searle A, Kirkup L. A direct comparison of wet, dry and insulating bioelectric recording electrodes. Physiol Meas. 2000;21:271-283.

8. Chang S, Ryu Y, Seunghwan K. Rubber electrode for wearable health monitoring. 2005 IEEE Engineering in Medicine and Biology 27th Annual Conference, Shanghai, China, 2005 Sep 1-4.

9. Muhlsteff J, Such O. Dry electrodes for monitoring of vital signs in functional textiles. Conf Proc IEEE Eng Med Biol Soc. 2004;3:2212-2215.

10. Baba A, Burke MJ. Measurement of the electrical properties of ungelled ECG electrodes. International Journal of Biology and Biomedical Engineering. 2008;2:89-97.

11. Lobodzinski SM, Laks MM. Biopotential fiber sensor. J Electrocardiol. 2006;39:S41-S46.

12. Paradiso R, Rossi DD. Advances in textile technogies for unobtrusive monitoring of vital parameters and movements. In proceedings of 28th IEEE EMBS Annual International Conference. New York, NY; 2006, Aug 30-Sep 3. Available at: http://embc2006.njit.edu/specialsymposiums.php Accessed on May 11, 2010.

13. Catrysse M, Puers R, Hertleer C, et al. Fabric sensors for measurement of physiological parameters. In: Proceedings of 12 th International Conference on Solid State Sensors, Actuators and Microsystems. Boston, MA. 2003;2;1415-1418.

14. Paradiso R, Loriga G, Taccini N. A wearable health care system based on knitted integrated sensors. IEEE Transactions on Information Technology in Biomedicine. 2005;9:337-344.

15. Prutchi D, Norris M. Design and Development of Medical Electronic Instrumentation. New York, NY: Wiley; 2005.

16. Levinzon LA. Ultra-low-noise high-input impedance amplifier for lowfrequency measurement applications. IEEE Transactions on Circuits and Systems. 2008;55:1815-1822.

17. Burr-Brown. INA116 data sheet, 2008. Available from: http://www. futurlec.com/BurrBrown/INA116.shtml Accessed on May 11, 2010.

18. Gargiulo G, Bifulco P, Calvo RA, Cesarelli M, Jin C, van Schaik AV. A mobile EEG system with dry electrodes. Abstract: Biomedical Circuits and Systems Conference, 2008. BioCAS 2008. Available from: http:// ieeexplore.ieee.org/xpl/freeabs_all.jsp?arnumber=4696927 Accessed on May 11, 2010. 
19. Bifulco P, Gargiulo G, Romano M, Fratini A, Cesarelli M. Bluetooth portable device for continuous ECG and patient motion monitoring during daily life. IFMBE Proceedings. 2007;16:369-372.

20. Corscience Bluesense AD data sheet V 1.2. 2006. Available from: http://www.corscience.de/en/medical-engineering/oemodm-solutions/ oem-modules/bluesense-easy.html Accessed on May 11, 2010.

21. Gargiulo G, Bifulco P, Calvo RA, Cesarelli M, Jin C, Van Schaik A. Mobile biomedical sensing with dry electrodes. Presented at: Fourth International Conference on Intelligent Sensors, Sensor Networks and Information Processing. 2008 Dec 15 -17, Sydney, Australia.
22. Artz W. Silicone-rubber electrodes for long-term patient monitoring. Biomed Eng. 1970;5:300-301.

23. Horowitz P, Hill W. The Art of Electronics. Cambridge: Cambridge University Press; 2002.

Medical Devices: Evidence and Research

\section{Publish your work in this journal}

Medical Devices: Evidence and Research is an international, peerreviewed, open access journal that focuses on the evidence, technology, research, and expert opinion supporting the use and application of medical devices in the diagnosis, treatment and management of clinical conditions and physiological processes. The identification of novel devices and optimal use of existing devices which will lead to improved clinical outcomes and more effective patient management and safety is a key feature. The manuscript management system is completely online and includes a quick and fair peer-review system. Visit http://www. dovepress.com/testimonials.php to read real quotes from authors.

Submit your manuscript here: http://www.dovepress.com/medical-devices-evidence-and-research-journal 